\title{
Modelo de Contorno Ativo Crisp: nova técnica de segmentação dos pulmões em imagens de TC
}

\author{
Pedro Pedrosa Rebouças Filho*, Paulo César Cortez, Marcelo Alcântara Holanda
}

Resumo Este trabalho propõe um novo método de contornos ativos (MCA), chamado de MCA Crisp, e o avalia na segmentação dos pulmões em imagens de Tomografia Computadorizada (TC). O MCA consiste em traçar uma curva inicial em torno ou dentro de um objeto de interesse. Esta curva se deforma, conforme algumas energias que atuam sobre a mesma, deslocando-a até as bordas do objeto. Este processo é realizado por iterações sucessivas de minimização de uma dada energia, associada à curva. Aplicando MCAs descritos na literatura na segmentação dos pulmões em imagens de TC, constatam-se limitações. Neste contexto, propõe-se o MCA Crisp para suprir tais limitações, propondo uma inicialização automática e uma nova energia externa baseada em regras e nas densidades radiológicas pulmonares. Realiza-se uma comparação entre os MCAs Tradicional, Balão, GVF e o método proposto para demonstrar a superioridade do novo método. Em seguida, para validar o MCA Crisp realiza-se uma análise qualitativa junto a um médico especialista na área de Pneumologia do Hospital Universitário Walter Cantídio da Universidade Federal do Ceará. Nesta análise, são utilizados 100 pulmões em imagens de TC. A eficiência da segmentação foi avaliada em 5 categorias, obtendo os seguintes resultados: 73\% ótimas, sem falhas, 20\% aceitáveis, com pequenos erros, 7\% razoáveis, com erros grosseiros e $0 \%$ ruim, segmentando apenas uma pequena parte do pulmão, e $0 \%$ péssima, obtendo uma segmentação totalmente errada. Conclui-se que o MCA Crisp é um método útil para segmentar os pulmões em imagens de TC e com potencial para integrar sistemas que auxiliem o diagnóstico médico.

Palavras-chave: Auxílio ao diagnóstico médico, Densidade radiológicas, Método de Contorno Ativo, Segmentação do pulmão, Tomografia Computadorizada do tórax.

\section{Active Contour Modes Crisp: new technique for segmentation the lungs in CT images}

\begin{abstract}
This paper proposes a new Active Contour Model (ACM), called ACM Crisp, and evaluates the segmentation of lungs in computed tomography (CT) images. An ACM draws a curve around or within the object of interest. This curve changes its shape, when some energy acts on it and moves towards the edges of the object. This process is performed by successive iterations of minimization of a given energy, associated with the curve. The ACMs described in the literature have limitations when used for segmentations of CT lung images. The ACM Crisp model overcomes these limitations, since it proposes automatic initiation and new external energy based on rules and radiological pulmonary densities. The paper compares other ACMs with the proposed method, which is shown to be superior. In order to validate the algorithm a medical expert in the field of Pulmonology of the Walter Cantídio University Hospital from the Federal University of Ceará carried out a qualitative analysis. In these analyses 100 CT lung images were used. The segmentation efficiency was evaluated into 5 categories with the following results for the ACM Crisp: 73\% excellent, without errors, 20\% acceptable, with small errors, and $7 \%$ reasonable, with large errors, $0 \%$ poor, covering only a small part of the lung, and 0\% very bad, making a totally incorrect segmentation. In conclusion the ACM Crisp is considered a useful algorithm to segment CT lung images, and with potential to integrate medical diagnosis systems.
\end{abstract}

Keywords: Medical diagnostic, Radiological densities, Active Contour Models, Lung Segmentation, Computerized tomography of the chest. 


\section{Extended Abstract}

\section{Introduction}

In order to prevent, detect and follow up diseases in the field of Pulmonology it is important to develop specialized tools for medical diagnosis, especially for the analysis of CT lung images. The development of segmentation techniques for CT lung images may accelerate and enhance the analytical diagnosis of a pulmonologist because currently this process is subjective and there is a great intra and interindividual variability.

Certain pulmonary diseases, may be detected and diagnosed early using CT images and even save lives in some cases. Furthermore, some techniques allow the clinical evolution of a disease to be followed up (Félix, 2007).

Among the most modern segmentation techniques that have a great potential for the development of new technologies is the Active Contour Models (ACM). This method was first proposed by Kass et al. (1987), and enables the segmentation of objects in digital images with a curve that distorts through information about its geometry and of the analyzed image.

This present paper proposes a new Active Contour Model, called ACM Crisp, capable of automatically segmenting the CT lung images. This method arises from the limitations found in the other potential methods described in the literature such as the Traditional, Balloon and Gradient Vector Flow (GVF) ACMs.

\section{Materials and Methods}

The new algorithm, called ACM Crisp, aims to overcome the limitations of the other ACMs for the segmentation of CT lung images. It is found that the ACMs of the literature, stabilize the edges closer to startup, by minimizing the energy through a local minimum. However, these edges are not exactly the pulmonary edges, but internal structures of the lungs.

To solve this problem, the new ACM Crisp method proposes initiating an automatic contour inside the lung and a new external force to be incorporated into the ACM Balloon developed by Mackiewich (1995). This is because the ACM Balloon produces promising results for the segmentation of CT lung images when initiated inside the lung borders. Also, its processing is faster than the GVF method. Thus, the mathematic formula for the new method is given by

$E_{\text {Crisp }}[c(s)]=w_{\text {cont }} F_{\text {cont }}[c(s)]+w_{\text {bal }} F_{b a l}[c(s)]+w_{\text {crisp }} F_{\text {crisp }}[c(s)](1)$

where $F_{\text {cont }}[c(s)]$ is the strength of continuity, which is the same as in the Traditional ACM (Kass et al., 1987), $F_{b a l}[c(s)]$ is the Balloon strength described by Mackiewich (1995) and $F_{\text {crisp }}[c(\mathrm{~s})]$ is the new force proposal by the ACM Crisp method.. The terms $w_{\text {cont }}, w_{\text {bal }}$ and $w_{\text {crisp }}$ are related to the weights of each force in the ACM Crisp energy composition.

This new strength, present in the ACM Crisp, is based on specific knowledge of pulmonary densities obtained from CT images. According to Gevenois and Yernault (1995) and Félix (2007), the pulmonary structures have specific values of radiological density measured in Hounsfield Units (HU). Thus, this force identifies which structures that are found by the operator Sobel, are the edges of the lung.

This is possible by analyzing the neighborhood of each edge using the radiological densities that is applied in this paper a $9 \times 9$ mask. To determine the intensity of the Crisp force at all points of the image, the percentage of each region of the image must be calculated first. For this, it is assumed that there are a set of six classes $s_{i}$, where i ranges from 0 to 5 , in the following order: 0 for hyper-airflow, 1 for normally airflow, 2 for slight airflow, 3 for non-airflow, 4 for bone and 5 for non classified areas. The definition of these zones is defined by the range of each region from the upper and lower limits of each structure shown in Figure 5.

In this sense, since $D(x, y)$ an array of lung densities, $(x, y)$ coordinates in which the neighborhood is analyzed, has the function that determines the amount of points with densities present in each class $S_{i}$ is determined by

$$
f\left(x, y, S_{i}\right)=\sum_{l=-4}^{4} \sum_{m=-4}^{4} R(x-l, y-m)
$$

where

$$
R(x, y)=\left\{\begin{array}{l}
1, \lim _{\text {inf }}\left(s_{i}\right)<D(x, y)<\lim _{\text {sup }}\left(s_{i}\right) \\
0, \text { otherwise }
\end{array}\right.
$$

where $\lim _{\text {inf }}\left(s_{i}\right)$ and $\lim _{\text {sup }}\left(s_{i}\right)$ represent the lower and upper range of density, described in $\mathrm{HU}$, for the class $\left(\mathrm{s}_{\mathrm{j}}\right)$.

When calculating the percentages of each class $s_{i}$ ignores the class 5 , since the areas not classified should not be included the calculation, therefore eliminating any noise. Thus, the percentage $P_{i}$ of each class $i$ is defined by

$$
P_{i}=\frac{f\left(x, y, s_{i}\right)}{\sum_{j=0}^{4} f\left(x, y, s_{j}\right)}
$$

Thus, considering the result of the Sobel operator on an array of densities $D(x, y)$ is a matrix $S(x, y)$, one has to force Crisp $F_{\text {crisp }}(x, y)$ is given by

$$
\mathrm{F}_{\text {Crisp }}(\mathrm{x}, \mathrm{y})=\left\{\begin{array}{l}
0, \text { para } \mathrm{P}_{3}<0,2 \text { e } \mathrm{P}_{4}<0,15 \\
\mathrm{~S}(\mathrm{x}, \mathrm{y}) \text {, otherwise }
\end{array}\right.
$$

The application of this equation defines the force Crisp $F_{\text {crisp }}(x, y)$, considering the Sobel gradient image, as shown in Figure 5.

Analyzing the Crisp forces $F_{\text {crisp }}(x, y)$ shown in Figures $5 j, k, l$, it was found that the energy from the internal structures of the lungs, shown in red in Figures 5g,h,i, are excluded, resulting in an external force with information only from the lung edges. Furthermore, this method proposed initialization, adding and removing of the curve points.

\section{Results and Discussion}

The proposed method was applied to HRCT images of patients with COPD and healthy volunteers. To assess the effectiveness of the proposed ACM method, the results were divided into three stages: analysis of the algorithm of initialization, comparative analysis, between the ACM Crisp and the other ACMs studied and the quality evaluation by the pulmonologist.

The analysis of the initialization algorithm was considered satisfactory, since all the curves were initialized within the lungs correctly. Initializations at the basis, hilus and apexis positions are shown in Figures $7 a, b, c$, respectively.

For the comparative analysis between the other ACMs studied and the Crisp method, the results obtained by ACMs Balloon and GVF are shown in the second and third line of Figure 7. In this case, the inefficacy of these methods to segment the CT lung images is noted. In contrast, the results obtained using the proposed method revealed to be effective in pulmonary segmentation, as shown in the fourth line of Figure 7.

The evaluation adopted in this paper uses the expertise of a pulmonologist to evaluate the results qualitatively, and this classification is presented in Table 1, and the results are shown in Table 2.

\section{Conclusions}

This paper proposes a new active contour method, called ACM Crisp, to automatically segment CT lung images. It also carries out a comparison of the Traditional, GVF and Balloon ACMs, 
using a set of 100 computed tomography images. HRCT images of healthy volunteers and patients with COPD were used. The segmentations by the proposed method were evaluated by $a$ pulmonologist from the Walter Cantídio University Hospital at the Federal University of Ceará, and 57\% of them were excellent segmentations, $36 \%$ were acceptable segmentations and $7 \%$ were reasonable segmentations. There were no bad or very bad segmentations. Thus, it can be said that 93\% of the segmentations obtained using the ACM Crisp method were satisfactory, and small errors could be corrected manually by the physician. However, the Traditional, Balloon and GVF ACMs presented unsatisfactory results, producing very bad segmentations in all CT lung images.

Therefore the Active Contours Models Crisp may be considered a useful algorithm for the segmentation of lung images and could be integrate to systems that assist medical diagnosis due to its satisfactory and automatic segmentation results. The time required to analyze the images was decreased, even in the images that had segmentation errors where some points needed to be corrected manually, without the necessity to make the complete segmentation, which is a tiresome and arduous operation. 


\section{Introdução}

Para prevenir, detectar e acompanhar doenças na área da Pneumologia é importante desenvolver ferramentas que auxiliem o diagnóstico do médico especialista, especificamente na análise de imagens de TC dos pulmões. Neste sentido, o desenvolvimento de técnicas de segmentação dos pulmões em imagens de TC pode acelerar e melhorar a eficácia da análise destes pelo médico pneumologista visto que atualmente este processo é subjetivo e envolve uma grande variabilidade intra e inter individual.

No processo de segmentação dos pulmões é importante que esta seja precisa, não podendo conter erros, pois estes podem resultar em diagnósticos imprecisos. Vale ressaltar que a segmentação manual demanda muito tempo, e é um processo enfadonho. Outro ponto importante é o aumento da precisão do diagnóstico do médico especialista à medida que aumenta o número de informações a sua disposição sobre o estado do paciente.

Neste sentido, é necessário desenvolver um método que possibilite uma segmentação correta, automática e precisa dos pulmões em imagens de TC do tórax. Dentre as técnicas de segmentação mais atuais que apresentam um grande potencial para o desenvolvimento de novas tecnologias, destaca-se o Método de Contorno Ativo (MCA). Este método proposto por Kass et al. (1987) permite realizar a segmentação de objetos em imagens digitais por meio de uma curva que se deforma através de informações de sua geometria e da imagem analisada.

O presente trabalho propõe um novo Método de Contorno Ativo, denominado MCA Crisp, capaz de segmentar automaticamente os pulmões em imagens de TC. Este método surge a partir das limitações encontradas nos MCAs descritos na literatura: Tradicional, Balão e Gradient Vector Flow (GVF).

\section{Materiais e Métodos}

O MCA foi introduzido por Kass et al. (1987), permitindo realizar a segmentação de objetos em imagens digitais por meio da detecção de bordas obtidas por informações da imagem. O MCA consiste em traçar uma curva inicial em torno ou dentro de um objeto de interesse. Esta curva se deforma, conforme algumas forças que atuam sobre a mesma, deslocando-se até as bordas do objeto. Este processo é realizado por iterações sucessivas de minimização de uma dada energia, associada à curva (Amini et al., 1990; Souza Júnior, 2006). O método proposto baseia-se nos MCAs descritos na literatura, principalmente nos métodos Tradicional, Balão e GVF.

\section{MCA tradicional}

Os contornos ativos são baseados em métodos variacionais, considerados modelos deformáveis, em que os pontos da curva são atualizados a cada nova iteração (Bouhours, 2006). Este modelo é chamado deformável porque está descrito por uma função de energia $E$ que varia segundo a Equação 1 (Kass et al. 1987)

$\mathrm{E}=\int_{0}^{1}\left\{\mathrm{E}_{\text {int }}[\mathrm{c}(\mathrm{s})]+\mathrm{E}_{\text {ext }}[\mathrm{c}(\mathrm{s})]\right\} \mathrm{ds}$

em que o termo $E_{\text {int }}$ representa as energias internas da curva, $E_{\text {ext }}$ o termo da energia associado com as energias externas e $c$ a curva, sendo que sua parametrização geométrica 2D é dada por (Kass et al., 1987)

$\left\{\begin{array}{l}{[0,1] \rightarrow \mathbb{R}^{2}} \\ s \rightarrow c(s)=[x(s), y(s)]\end{array}\right.$

no qual a posição de cada ponto $c(s)$ na imagem é determinada pelas coordenadas $x(s)$ e $y(s)$.

A energia interna é proveniente da geometria da curva, relacionada com sua forma e a posição de seus pontos (Itai et al., 2005). A energia externa é proveniente das características da imagem e está associada às informações obtidas a partir desta (Kass et al., 1987).

A energia externa $E_{\text {ext }}$ geralmente é obtida a partir de um operador gradiente, dentre os quais o Sobel é o mais utilizado (Nixon e Aguado, 2002). Já a energia interna $E_{\text {int }}$ é descrita por (Xu e Prince, 1997)

$E_{\text {int }}(s)=\alpha(s)\left|\frac{d c(s)}{d s}\right|^{2}+\beta(s)\left|\frac{d^{2} c(s)}{d s^{2}}\right|^{2}$

em que $\alpha(s)$ especifica o valor do peso da força interna que define a elasticidade da curva, enquanto $\beta(s)$ atribui o valor do peso da força interna que caracteriza a resistência da curva à torção. $\mathrm{O}$ termo $\left|\frac{d c(s)}{d s}\right|$ tende a espaçar igualmente os pontos, já o termo $\left|\frac{d^{2} c(s)}{d s^{2}}\right|$ tende a suavizar a curvatura da curva.

De acordo com Cohen (1991) e Mackiewich (1995), o método Tradicional não detecta a borda da imagem quando é inicializado longe desta, ou seja, quando a borda não está presente na zona determinada pela vizinhança de cada ponto. Neste contexto, são propostos dois MCAs que superam tal limitação, o MCA Balão (Mackiewich, 1995) e o Gradient Vector Flow (Xu e Prince 1997).

\section{MCA balão}

O MCA Balão propõe uma nova força interna denominada força Balão. Esta força Balão é utilizada 
para expandir a curva devido ao fato de que, em regiões homogêneas da imagem, a força externa determinada pelo gradiente é nula, então as forças que interferem no contorno são referentes às forças internas.

A Força Balão deforma o contorno, a partir de uma informação conhecida a priori, para este se aproximar das bordas. Dentre os tipos de informação conhecidas, Mackiewich (1995) assume que a curva é inicializada dentro do objeto de interesse, e minimiza esta força afastando o contorno do baricentro da curva.

\section{MCA gradient vector flow}

O principal problema no uso do gradiente de uma imagem como uma força externa no MCA é que esta energia só possui informações nas bordas desta imagem, sendo nulo em regiões homogêneas da imagem (Xu e Prince, 1997).

De acordo com Xu e Prince (1997), o MCA Gradient Vector Flow (GVF) constrói um campo de vetores que direciona o fluxo gradiente para as bordas da imagem, conduzindo a curva até as bordas do objeto. A energia $E$ deste MCA é dada pela equação

$$
E=\alpha(s)\left|\frac{d c(s)}{d s}\right|^{2}+\beta(s)\left|\frac{d^{2} c(s)}{d s^{2}}\right|^{2}+E_{e x t}[c(s)]
$$

em que as energias internas $\left|\frac{d c(s)}{d s}\right|$ e $\left|\frac{d^{2} c(s)}{d s^{2}}\right|$ são as mesmas do MCA Tradicional, sendo $\alpha(s)$ e $\beta(s)$ seus respectivos pesos. Já a energia externa $E_{\text {ext }}$ é a expansão do mapa das bordas da imagem proveniente de um operador gradiente, e esta é definida por (Xu e Prince, 1997)

$$
E_{e x t}=\iint\left\{\mu\left(\frac{d^{2} u}{d x}+\frac{d^{2} u}{d y}+\frac{d^{2} v}{d x}+\frac{d^{2} v}{d y}\right)+|\nabla f|^{2}+|\omega-\nabla f|^{2}\right\} d x d y
$$

em que $\frac{d^{2} \mathrm{u}}{d x}, \frac{d^{2} \mathrm{u}}{d y}, \frac{d^{2} \mathrm{v}}{d x}$ e $\frac{d^{2} \mathrm{v}}{d y}$ são as derivadas parciais de $u$ e $v$, em relação aos eixos $x$ e $y$, respectivamente.
O parâmetro $\mu$ permite ajustar o equilíbrio entre os dois termos da Equação 5.

O campo de Fluxo Gradiente em uma imagem depende diretamente da parametrização do fator $\mu$ da Equação 5 e do número de iterações da aplicação desta mesma equação na imagem. $\mathrm{O}$ valor ótimo do fator $\mu$ sugerido por Bouhours (2006) é 0,1. Sendo assim, aplicando a Equação 5 em uma imagem com 100, 200 e 400 iterações, conforme ilustrado na Figura 1 constata-se que a área ocupada pelo fluxo de vetores gradiente aumenta proporcionalmente ao número de iterações.

\section{Metodologia}

Nesta seção é apresentada a forma de aquisição das imagens digitais através de Tomografia Computadorizada. Em seguida, os MCAs citados são aplicados na segmentação dos pulmões em imagens de TC e suas limitações são discutidas. Por fim, um novo MCA é proposto buscando suprir tais limitações.

\section{Aquisição das imagens}

Neste processo de aquisição de imagens é utilizado uma Tomografia Computadorizada de Alta Resolução (TCAR). Estas imagens são adquiridas em um tomógrafo Toshiba modelo Auklet com a espessura da fatia de corte de $1,5 \mathrm{~mm}$, ajuste da janela pulmonar: centro e largura, respectivamente de -600\1600 UH, a dimensão da imagem reconstruída é de $512 \times 512$ pixels, e voxel com dimensões $0,585 \times 0,585 \times 1,5 \mathrm{~mm}$. As imagens são quantificadas em 16 bits e armazenadas no padrão DICOM (Digital Imaging and Communications in Medicine).

Estas imagens foram obtidas em parceria com o Hospital Walter Cantídio da Universidade Federal do Ceará, cedidas por um estudo anterior (Fortaleza, 2006; Winkeler, 2006). Este estudo foi avaliado e aprovado
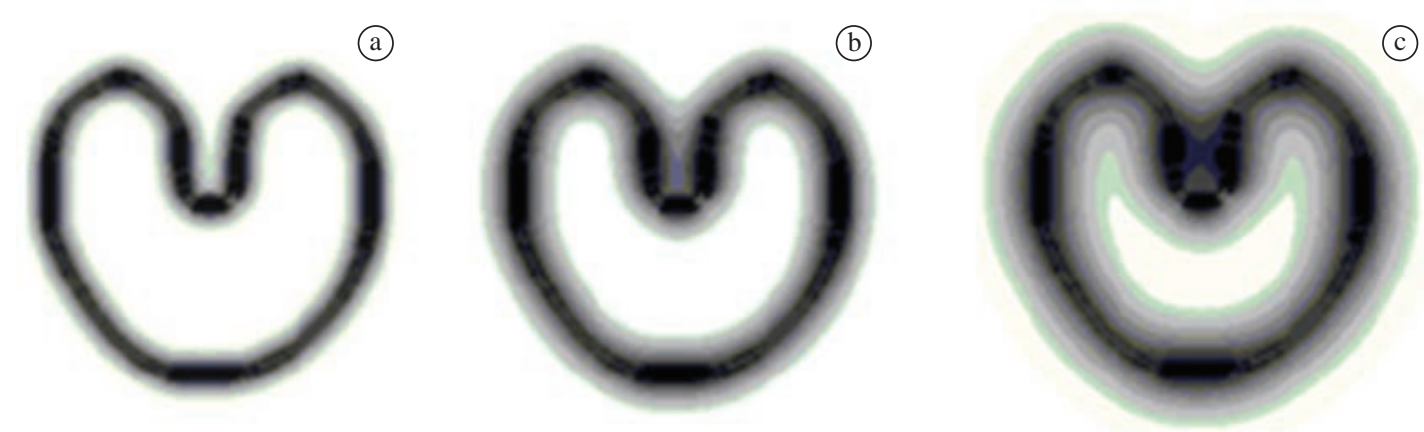

Figura 1. Fluxo de vetores gradiente obtido com a) 100 iterações, b) 200 iterações e c) 400 iterações. Figure 1. Gradient vector flow obtained with a) 100 iterations, b) 200 iterations and c) 400 iterations. 
pelo Comitê de Ética em Pesquisa da UFC - COMEPE (Protocolo $\left.n^{\circ} 35 / 06\right)$ e incorporado às exigências da Resolução nº 196/96 do Conselho Nacional de Saúde, a respeito de pesquisas em seres humanos.

As imagens são obtidas a partir de voluntários sadios e de pacientes com Doença Pulmonar Obstrutiva Crônica (DPOC). Além destas imagens obtidas neste estudo anterior (Fortaleza, 2006; Winkeler, 2006), foram utilizadas imagens de Tomógrafo Multidetector cedidas por um voluntário sadio visando testar a eficácia dos métodos em tomógrafos distintos.

Para a leitura destas imagens é utilizada a biblioteca livre DCMTK (Dicom ToolKit ) fornecida pela OFFIS. Esta biblioteca é compatível com a linguagem de programação $\mathrm{C}++$, a mesma utilizada no desenvolvimento deste trabalho.

Cada estrutura interna do corpo humano possui um determinado valor de densidade radiológica, expresso em Unidades Hounsfield. Esta densidade é obtida pelo tomógrafo emitindo feixes de raio- $\mathrm{X}$ no corpo humano, e cada tecido deste possui um coeficiente de atenuação linear distinto. Deste modo, cada tecido do corpo humano possui uma densidade radiológica específica. As faixas de densidade radiológica pulmonar adotadas neste trabalho são: áreas hiperaeradas ou hiperinsufladas $(-1000$ a $-950 \mathrm{UH})$, normalmente aerada (-950 a $-500 \mathrm{UH})$, pouco aerada ( -500 a $-100 \mathrm{UH})$, não aerada (-100 a $100 \mathrm{UH})$ e região óssea (600 a 2000 UH) (Gevenois e Yernault, 1995; Félix, 2007). Na Figura 2b é mostrado um exemplo desta distribuição, em que a cor vermelha representa regiões hiperaeradas, azul escuro a normalmente aerada, azul claro representa as áreas pouco aeradas, preto a não aerada e verde o osso, sendo a imagem original apresentada na Figura 2a.

\section{Limitações dos MCAs tradicional, balão e GVF na segmentação dos pulmões em imagens de TC}

Após descrever os MCAs Tradicional, Balão e GVF é possível avaliar o desempenho de cada um na segmentação de imagens de TC dos pulmões.

No processo de segmentação dos pulmões em imagens de TC de um modo geral, a primeira limitação é a inicialização da curva fora dos pulmões, devido a quantidade de formas possíveis dos pulmões, seja pela escolha do nível de corte da visualização no tomógrafo ou pelas diferenças das estruturas dos pulmões entre seres humanos, conforme são mostradas nas Figuras 3a,b,c os níveis base, ápice e hilo, respectivamente.

Além disto, existe a presença de ar dentro e fora dos pulmões nas regiões superior e inferior da imagem, confundindo com as estruturas dos pulmões, conforme
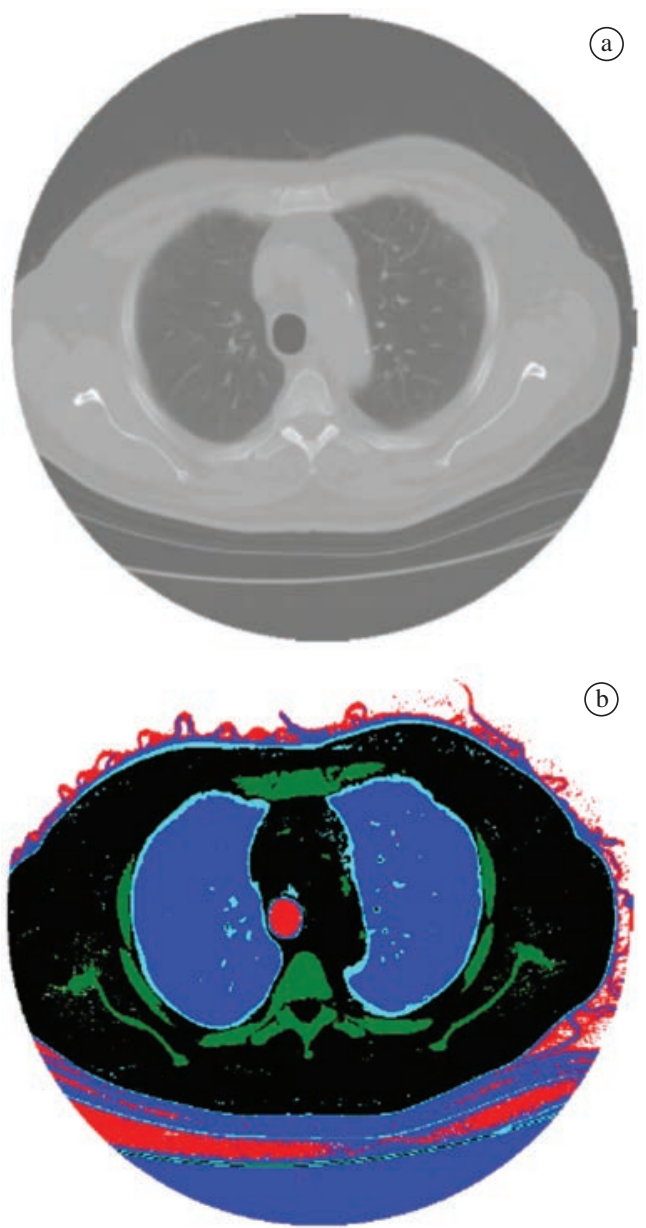

Figura 2. Imagem de TC dos pulmões: a) obtida com TCAR e b) representação das faixas de densidades.

Figure 2. CT image of the lungs: a) obtained with HRCT and b) representation of bands of densities.

é mostrado na Figura 2b. Outro ponto importante é que as densidades radiológicas da imagem podem ser alteradas seja por ruído gerado na aquisição das imagens ou por filtros aplicados na mesma. Sendo assim, a inicialização da curva dentro dos pulmões próximo das bordas e fora dos pulmões é inviável, impossibilitando a segmentação dos pulmões em imagens de TC utilizando o MCA Tradicional.

Na sua essência os MCAs Balão e GVF visam resolver o problema de inicialização da curva observado no MCA Tradicional, e para avaliar estes métodos na segmentação dos pulmões em imagens de TC é usada uma imagem tida como regular, ou seja, que possui uma forma com poucos detalhes e menos estruturas pulmonares (vasos, vias aéreas, etc), conforme é ilustrado na Figura 4a. Isto é usado para demonstrar a ineficácia destes métodos, mesmo em imagens regulares. 

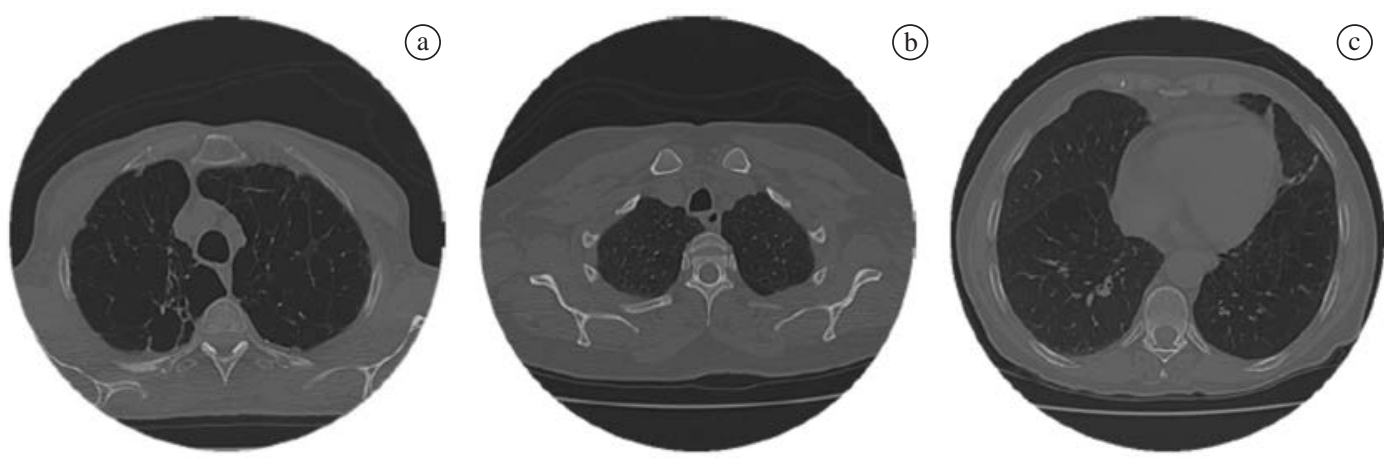

(d)
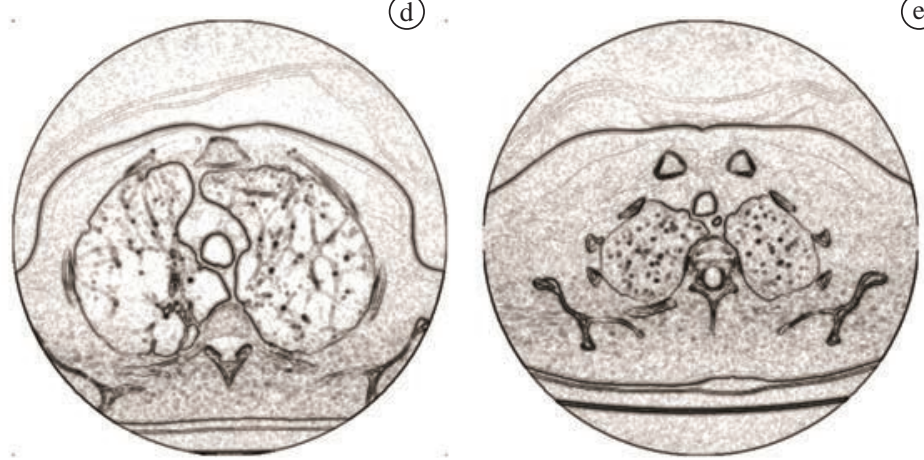

(e)

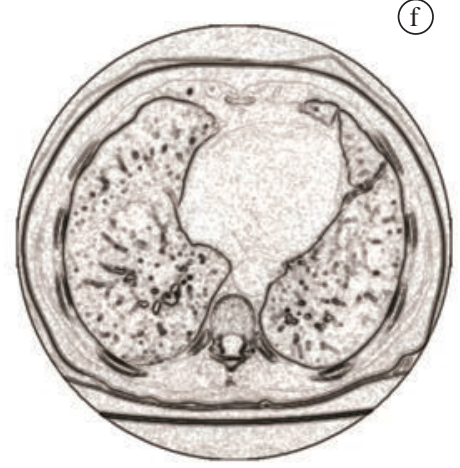

(g)
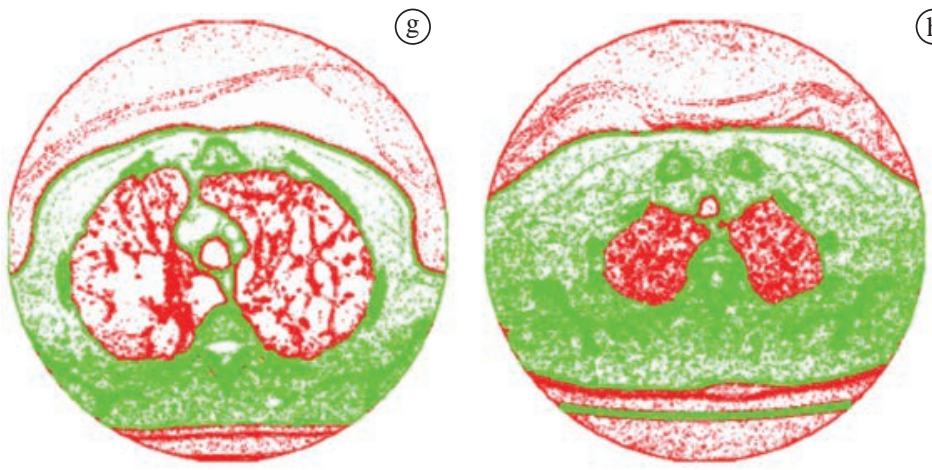

(h)

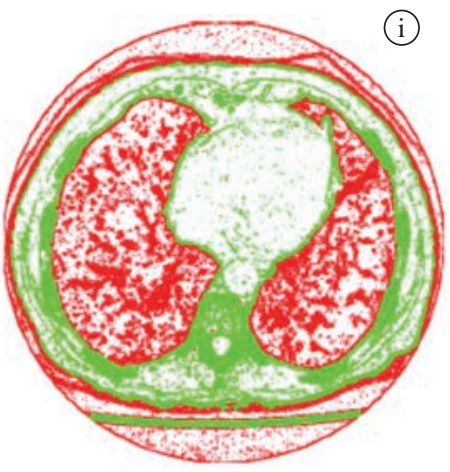

(k)

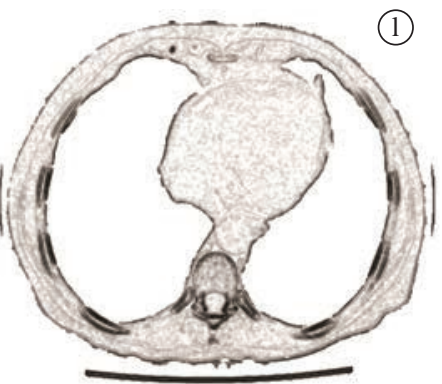

Figura 3. Imagem de TC dos pulmões: a,b,c) originais, d,e,f) gradiente Sobel, g,h,i) resultado da análise realizada pela força externa do método proposto, j,k,l) força externa do método proposto.

Figure 3. CT image of the lungs: $a, b, c)$ original, $d, e, f)$ Sobel gradient, $g, h, i)$ the result of analysis carried out by external force of the proposed method, $j, k, l)$ external force of the proposed method. 
A inicialização da curva é apresentada em vermelho no pulmão à esquerda na Figura 4a e os resultados dos métodos Balão e GVF são apresentados nas Figuras 4b,c, respectivamente.

Constata-se, por observar a Figura 4, a ineficácia destes métodos na segmentação do pulmão quando inicializado afastado das bordas. Isto ocorre devido a origem da energia externa, pois o MCA Balão utiliza como energia externa a energia tradicional sugerida por Kass et al. (1987). Já o método GVF expande as bordas encontradas na energia externa Tradicional. Deste modo, ambos os métodos atraem a curva até a borda mais próxima. Entretanto, esta energia não distingue as bordas do pulmão das bordas das estruturas internas do mesmo. Sendo assim, a curva é atraída para as bordas mais próximas. Exemplos destas energias utilizando a imagem original mostrada na Figura 5a são apresentadas nas Figuras 5b,c, respectivamente para o MCA Tradicional e para o MCA GVF utilizando $\mu$ igual a 0,1 e com 400 iterações.
Resumindo, constata-se que a segmentação dos pulmões em imagens de TC possui restrições devido aos seguintes fatores:

- Distorção das densidades radiológicas, seja por ruídos na aquisição das imagens através da TCAR, ou por filtros aplicados na imagem;

- Problemas com a inicialização da curva, podendo ocorrer por quatro motivos; primeiro, as imagens adquiridas em cada paciente são distintas e possuem tamanho e posicionamento diferentes; segundo, a visualização de um órgão depende da posição do corte analisado, onde um órgão pode assumir diferentes tamanhos e formatos; terceiro, existe ar dentro e fora dos pulmões, dificultando a identificação das densidades radiológicas das regiões internas do pulmão; quarto, a espessura da fatia da imagem é relevante visto que a densidade é retirada da média dos valores presentes em uma fatia, e quanto maior esta fatia, maior a distorção ou imprecisão da densidade;
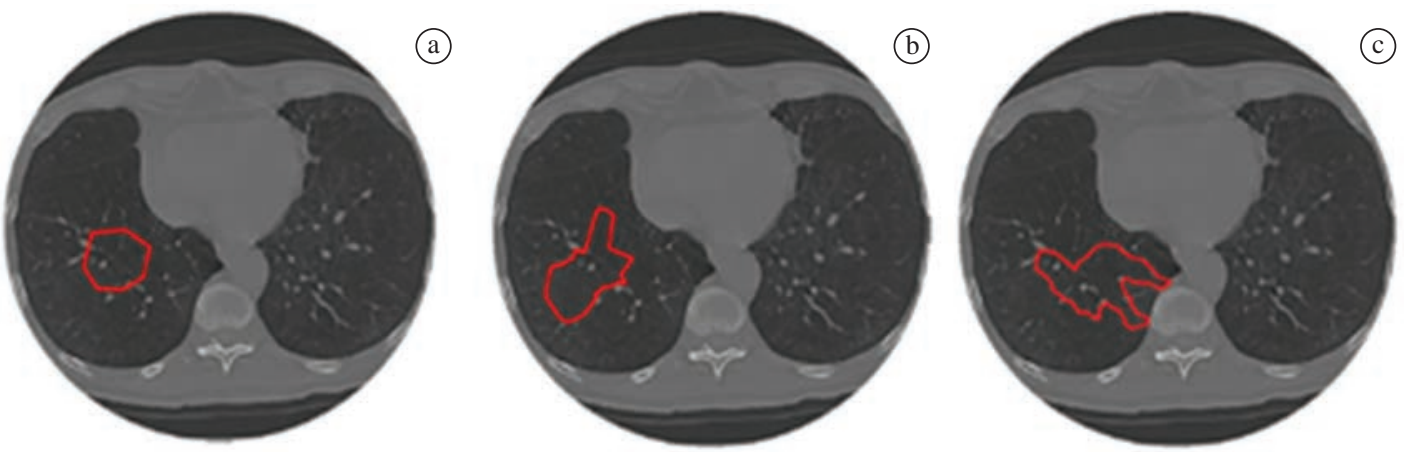

Figura 4. Imagens de TC do pulmão com: a) inicialização do MCA, b,c) resultado da segmentação obtida pelos MCAs Balão e GVF, respectivamente.

Figure 4. CT images of the lung: a) initiate the ACM, b,c) the result of segmentation obtained by ACMs balloon and GVF, respectively.
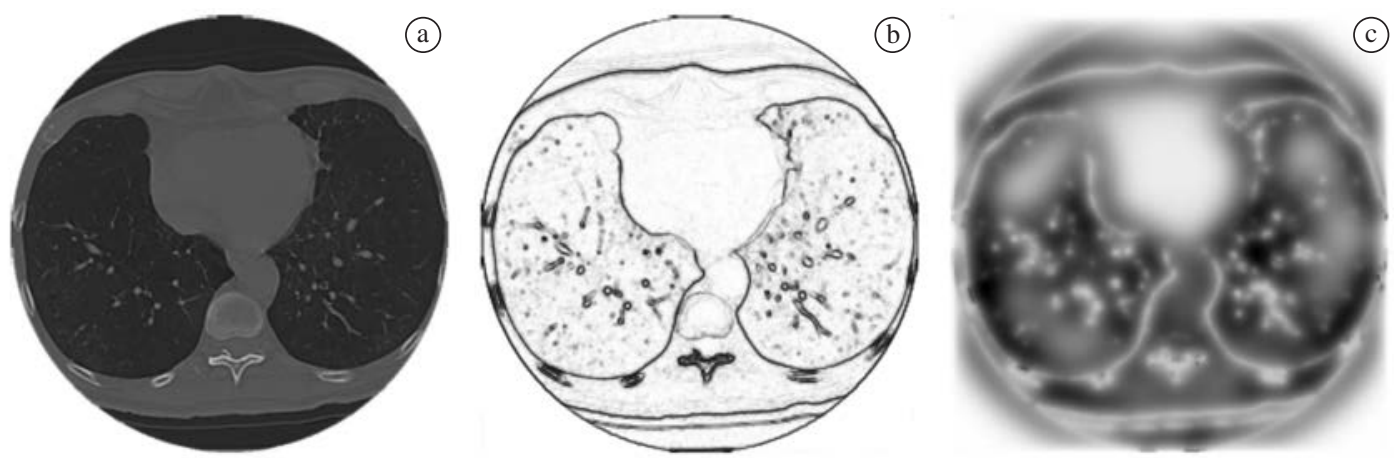

Figura 5.Imagens de TC dos pulmões: a) original e b) resultado do gradiente Sobel, c) resultado do vetor de fluxo gradiente com 400 iterações. Figure 5. CT images of the lungs: a) original and b) the results of gradient Sobel, c) the result of the gradient vector flow with 400 iterations. 
- Problemas com a detecção de falsas bordas na imagem, o que ocorre devido à detecção de objetos que não são o alvo da segmentação.

\section{Método de contorno ativo Crisp}

O novo algoritmo, intitulado MCA Crisp, surge visando superar as limitações observadas na aplicação dos MCAs na segmentação dos pulmões em imagens de TC. É constatado na seção anterior que os MCAs analisados, ao serem inicializados afastados das bordas, estabilizam-se após minimizar sua energia nas bordas encontradas (mínimos locais). Entretanto, estas bordas não são exatamente as bordas pulmonares, mas de estruturas internas dos pulmões.

Para solucionar esse problema, o novo método MCA Crisp propõe uma inicialização automática do contorno dentro do pulmão e uma nova força externa a ser incorporada ao MCA Balão desenvolvido por Mackiewich (1995). Isto porque este produz resultados promissores para o processo de segmentação dos pulmões em imagens de TC, quando inicializado afastado das bordas. Além disso, seu processamento é rápido comparado com o método GVF. Deste modo, a formulação matemática para o novo método é dada por

$$
E_{\text {Crisp }}[c(s)]=w_{\text {cont }} F_{\text {cont }}[c(s)]+w_{\text {bal }} F_{\text {bal }}[c(s)]+w_{\text {crisp }} F_{\text {crisp }}[c(s)]
$$

em que $F_{\text {cont }}[c(s)]$ é a força de continuidade, a mesma utilizada no MCA Tradicional citada na Equação 3, $F_{\text {bal }}[c(s)]$ é a Força Balão descrita por Mackiewich (1995) e $F_{\text {crisp }}[c(s)]$ é a nova força proposta pelo método MCA Crisp. Os termos $w_{\text {conte }}$, $w_{\text {bal }}$ e $w_{\text {crisp }}$ são referentes aos pesos de cada força na composição da energia do MCA Crisp, que neste artigo foi definido 0,1 para $w_{\text {cont }} 0,1$ para $w_{\text {bal }}$ e 0,8 para $w_{\text {crisp }}$. Esta parametrização valoriza a força Crisp para esta ter $80 \%$ da composição da Energia Crisp, enquanto as outras são menos significativas com apenas $10 \%$ para cada uma das outras forças.

Essa nova força, presente no MCA Crisp, é baseada no conhecimento específico das densidades pulmonares obtidas em imagens de TC. Segundo Gevenois e Yernault (1995) e Félix (2007), as estruturas pulmonares possuem valores específicos de densidade medidas em Unidades Hounsfield (UH). Deste modo, esta força identifica quais estruturas encontradas através do operador Sobel são as bordas do pulmão.

Isto é possível analisando a vizinhança de cada borda encontrada utilizando as densidades radiológicas do pulmão em imagens de TC. Observando tal vizinhança, percebe-se que as bordas de dentro do pulmão possuem um padrão diferente das bordas situadas fora do pulmão. O tamanho da máscara desta vizinhança deve ser maior que $3 \times 3$, considerando todos os pixels desta máscara no cálculo, pois menor que isso não se tem informações suficientes para observar os padrões pré-estabelecidos. Percebe-se que ao aumentar a máscara, aumenta-se a informação para análise, melhora os resultados e diminui a velocidade de processamento. Após avaliar o resultados de diversas dimensões de máscaras para análise desta vizinhança, percebe-se que as máscaras maiores que $9 \times 9$ não melhoram significativamente os resultados, além de diminuir a eficiência do método. Deste modo, a análise da vizinhança ótima adotada neste trabalho é a máscara $9 \times 9$.

Para se determinar a intensidade da Força Crisp em todos os pontos da imagem, primeiramente é necessário estabelecer uma função para calcular os percentuais de cada umas das regiões presentes na imagem. Para isto, assume-se que existem um conjunto de 6 classes $s_{\mathrm{i}}$, em que $i$ varia de 0 a 5 , na seguinte ordem: 0 para hiper-aerada (-1000 a $-950 \mathrm{UH})$, 1 para normalmente aerada ( -950 a $-500 \mathrm{UH}), 2$ para pouco aeradas ( $-500 \mathrm{a}-100 \mathrm{UH}), 3$ para não aerada ( -100 a $100 \mathrm{UH}), 4$ para osso (600 a $2000 \mathrm{UH})$ e 5 para áreas não classificadas. A definição destas regiões é realizada definindo a faixa de cada região a partir dos limites inferior e superior de cada estrutura demonstrada na Figura 2.

Neste sentido, considerando $D(x, y)$ uma matriz de densidades pulmonares, $(x, y)$ as coordenadas nas quais a vizinhança é analisada, tem-se que a função que determina a quantidade de pontos com densidades presente em cada classe $\mathrm{s}_{\mathrm{i}}$, é determinada por

$f\left(x, y, s_{i}\right)=\sum_{l=-4}^{4} \sum_{m=-4}^{4} R(x-l, y-m)$

no qual

$\mathrm{R}(\mathrm{x}, \mathrm{y})=\left\{\begin{array}{l}1, \lim _{\text {inf }}\left(\mathrm{s}_{\mathrm{i}}\right)<D(\mathrm{x}, \mathrm{y})<\lim _{\text {sup }}\left(\mathrm{s}_{\mathrm{i}}\right) \\ 0, \text { caso contrário }\end{array}\right.$

em que $\lim _{\text {inf }}\left(\mathrm{s}_{\mathrm{i}}\right)$ e $\lim _{\text {sup }}\left(\mathrm{s}_{\mathrm{i}}\right)$ representam os limites inferior e superior da faixa de densidade, descrita em UH, para a classe $\mathrm{s}_{\mathrm{i}}$.

Ao calcular os percentuais de cada classe $\mathrm{s}_{\mathrm{i}}$, desconsidera-se a classe 5 , visto que as áreas não classificadas não devem compor o cálculo. Logo, o percentual $\mathrm{P}_{\mathrm{i}}$ de cada classe $i$ é definido por

$P_{i}=\frac{f\left(x, y, s_{i}\right)}{\sum_{j=0}^{4} f\left(x, y, s_{j}\right)}$

Deste modo, considerando que o resultado do operador Sobel em uma matriz de densidades $D(x, y)$ seja uma matriz $S(x, y)$, tem-se que a força Crisp $F_{\text {Crip }}(x, y)$ é dada por

$\mathrm{F}_{\text {Crisp }}(\mathrm{x}, \mathrm{y})=\left\{\begin{array}{l}0, \text { para } \mathrm{P}_{3}<0,2 \text { e } \mathrm{P}_{4}<0,15 \\ \mathrm{~S}(\mathrm{x}, \mathrm{y}), \text { caso contrário }\end{array}\right.$ 
A aplicação desta equação define a força Crisp $F_{\text {Crisp }}(x, y)$ considerando o gradiente Sobel da imagem, conforme é demonstrado na Figura 3.

Analisando as Forças Crisp $F_{\text {Crisp }}(x, y)$ mostradas nas Figuras $3 \mathrm{j}, \mathrm{k}, \mathrm{l}$, percebe-se que as energias provenientes das estruturas internas dos pulmões, demonstradas em vermelho nas Figuras 3g,h,i, são excluídas, resultando em uma força externa apenas com informações das bordas dos pulmões. Neste sentido, para finalizar o MCA proposto faltam as etapas de inicialização da curva, adição e remoção de pontos da curva.

$\mathrm{O}$ algoritmo de inicialização do contorno tem como objetivo encontrar as coordenadas iniciais para este. No caso de imagens de TC dos pulmões, tem-se dois objetos de interesse, os pulmões direito e esquerdo. Assim, têm-se um ponto de inicialização para cada pulmão, sendo o ponto de inicialização esquerdo PIE e o ponto de inicialização direito PID. Para se determinar as coordenadas do PIE e do PID, necessitase de informação a priori. Analisando várias imagens de TC dos pulmões, observa-se que a imagem pode ser dividida nas regiões superior, inferior, esquerda e direita, separadas em vermelho na Figura 6.

Inicialmente, as regiões superior e inferior são excluídas da análise, visto que existe semelhança das densidades radiológicas do ar dentro dos pulmões e do ar das regiões inferior e superior, que são provenientes da região do tomógrafo não ocupada pelo corpo do paciente. Este artifício diminui o tempo de processamento e aumenta a taxa de acerto do método de inicialização.

O primeiro passo para a inicialização é identificar as densidades radiológicas da região interna do objeto de interesse, que no caso estudado consistem nas áreas hiperaeradas, com densidades entre -1000 e -950 UH, e nas áreas normalmente aeradas, com densidades entre -950 e -500 UH. Estas regiões estão mostradas em azul claro e escuro na Figura 2b, respectivamente.

O segundo passo é limitar cada região de inicialização, determinando os limites nos eixos das abscissas e das ordenadas. A partir disto, a linha que obtiver a maior incidência na faixa radiológica desejada $(-1000$ a $-500 \mathrm{UH})$, passa a ser a coordenada $y$ de inicialização. Do mesmo modo, a coluna que obtiver a maior incidência na faixa radiológica desejada, passa a ser a coordenada $x$ de inicialização. Após análise de diversas imagens, a faixa adotada neste trabalho para a região esquerda é 0-256 para o eixo x, e 180-360 no eixo y. Para a região direita a faixa do eixo y se mantém e a faixa do eixo x é 256-512. A região analisada considerando esses parâmetros está ilustrada Figura 6, em vermelho, assim como os pontos PIE e PID estão em amarelo.

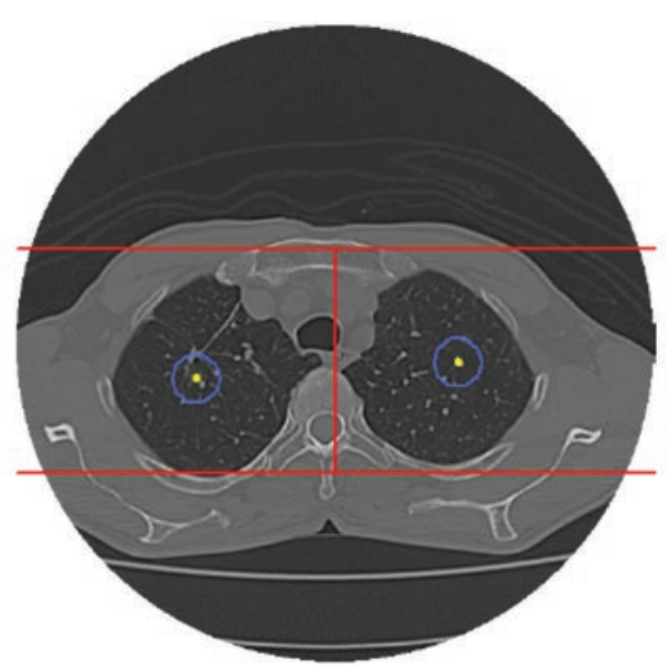

Figura 6. Demonstração das regiões utilizadas para encontrar os pontos para as inicializações dos contornos dos pulmões esquerdo e direito, em vermelho, em amarelo os pontos de inicialização e o contorno gerado em azul.

Figure 6. Demonstration of the regions used to find the points for initializations from the contour of the lungs left and right, in red, yellow points of initiating and contour generated in blue.

Os pontos PIE e PID, em amarelo na Figura 6, são utilizados como baricentro de um polígono regular. A distância de cada ponto para o baricentro é configurada no algoritmo de inicialização, juntamente com a quantidade de vértices do polígono. Deste modo, após testes exaustivos, foi utilizada uma distância de 10 pixels entre cada ponto e o baricentro, e um total de 20 vértices, conforme é apresentado, em azul, na Figura 6.

A inclusão e exclusão de pontos também são elementos importantes na dinâmica do MCA Crisp. A inclusão permite um maior refinamento da curva e, portanto, maior acurácia na segmentação dos contornos de objetos. A exclusão elimina pontos do contorno que estavam em discrepância com os seus vizinhos e elimina pontos vizinhos que possuem as mesmas coordenadas.

O método de adição de pontos se baseia na distância Euclidiana entre dois pontos consecutivos do contorno. Deste modo, quando esta distância é maior do que uma distância máxima entre pontos, um novo ponto é adicionado entre estes, aumentando a sensibilidade do contorno. Com base na análise de diversas imagens, é adotado o valor de 5 pixels como distância máxima.

Já o método de remoção de pontos baseia-se na remoção de pontos que possuem o ângulo formado entre o ponto analisado e seus vizinhos inferior a um ângulo mínimo. $\mathrm{O}$ valor de referência adotado neste artigo é $60^{\circ}$ como ângulo mínimo. Isto faz com 
que pontos que estão muito afastados do restante do contorno sejam apagados, diminuindo erros grosseiros. Após apresentar o método proposto, é necessário verificar sua eficácia comparando com outros métodos e, principalmente, junto ao médico especialista na área de pneumologia.

\section{Resultados e Discussão}

O método proposto foi aplicado em imagens de TCAR utilizando imagens de pacientes com DPOC e voluntários sadios. Neste sentido, para avaliar a eficácia do MCA proposto, os resultados obtidos são divididos em três etapas: análise do algoritmo de inicialização, análise comparativa entre o MCA Crisp e os outros MCAs estudados e avaliação qualitativa junto ao médico pneumologista.

\section{Análise do algoritmo de inicialização}

Na análise do algoritmo de inicialização foi avaliada a eficácia do método de inicialização, visto que a curva deve ser inicializada dentro do pulmão. Quando o algoritmo inicializa a curva dentro dos pulmões, esta inicialização é dita satisfatória.

Nesta etapa de resultados, são utilizadas 50 imagens entre pacientes com DPOC e voluntários sadios, nas posições ápice, hilo e base obtidas a partir de imagens de TCAR e de TC com multidetectores, totalizando 100 pulmões em imagens de TC, visto que cada imagem possui dois pulmões. Algumas destas inicializações são mostradas nas Figuras 7a,b,c,d.

Os resultados obtidos com esta inicialização nas imagens estudadas são considerados satisfatórios, visto que todas as curvas são inicializadas dentro dos pulmões corretamente.

\section{Análise comparativa entre os MCAs Crisp, Balão e GVF}

Para a análise comparativa entre os MCAs estudados e o Crisp, resultados para diferentes imagens são apresentados, duas imagens de pacientes com DPOC, Figuras 7a,b, uma de um voluntário sadio, Figura 7c, obtidas no TCAR e uma de voluntário sadio obtida no tomógrafo com multidetectores, Figura 7d. Nestas mesmas figuras são apresentadas, em vermelho, as inicializações utilizadas em todos os métodos.

Os resultados obtidos pelos MCAs Balão e GVF são demonstrados na segunda e terceira linha da Figura 7. Neste caso, verifica-se a ineficácia destes métodos na segmentação dos pulmões em imagens de TC. Em contrapartida, os resultados obtidos utilizando o método proposto revelaram-se eficazes na segmentação pulmonar, conforme é demonstrado na quarta linha da Figura 7. Os resultados obtidos com o tomógrafo com multidetectores foi equivalente aos obtidos no TCAR.

Analisando os resultados descritos na Figura 7, percebe-se a eficácia do método proposto na segmentação dos pulmões em imagens de TC, considerando imagens tomográficas de pulmões em diferentes níveis de corte e, portanto, com diferentes áreas, formas e tamanhos.

\section{Avaliação qualitativa do médico especialista}

No caso de validação de algoritmos de imagens médicas, alguns autores procedem utilizando a experiência de médicos especialistas. Zijdenbos et al. (1994) e Zaidi et al. (2010) validam algoritmos com base na comparação visual entre os resultados apresentados e segmentações manuais realizadas por médicos especialistas. Já Sardanelli et al. (2004) avalia o algoritmo com base na atribuição de notas de radiologistas aos resultados obtidos.

A avaliação adotada neste trabalho segue o mesmo princípio, utilizar a experiência de um pneumologista para avaliar os resultados de forma qualitativa. Dentre as avaliações qualitativas descritas na literatura, este trabalho utiliza uma avaliação qualitativa baseada na avaliação qualitativa de segmentação descrita por Gonzales e Woods (2008) que classifica a segmentação em classes, em que o critério é a qualidade da segmentação. Deste modo, são consideradas 5 classes: ótimo, aceitável, razoável, ruim e péssimo. Esta classificação está descrita na Tabela 1.

Seguindo o critério de avaliação estabelecido, pode-se constatar que os resultados obtidos pelos métodos Balão e GVF obtém segmentação ruim em todos os casos analisados na Figura 7. Já o método proposto obteve segmentações ótimas para as mesmas imagens.

Com o intuito de classificar o método proposto adequadamente, a avaliação dos resultados deste é realizada junto a um médico especialista na área da Pneumologia do Hospital Universitário Walter Cantídio da Universidade Federal do Ceará. Nesta etapa, são utilizadas 50 imagens entre 11 pacientes com DPOC e 8 voluntários sadios obtidas a partir de TCAR, totalizando 100 segmentações de pulmão em imagens de TC. Estas imagens foram adquiridas na posição supina, em que 20 são em nível de ápice, 15 em nível de hilo e 15 em nível de base. Na Tabela 2 é mostrado o resultado da avaliação realizada pelo médico especialista em cada nível separadamente e, em seguida, a análise geral obtida.

Com base nos resultados apresentados na Tabela 2, constata-se que em nível de ápice 97,5\% das segmentações são consideradas ótimas pelo médico especialista, e os $2,5 \%$ restantes possuem pequenos 

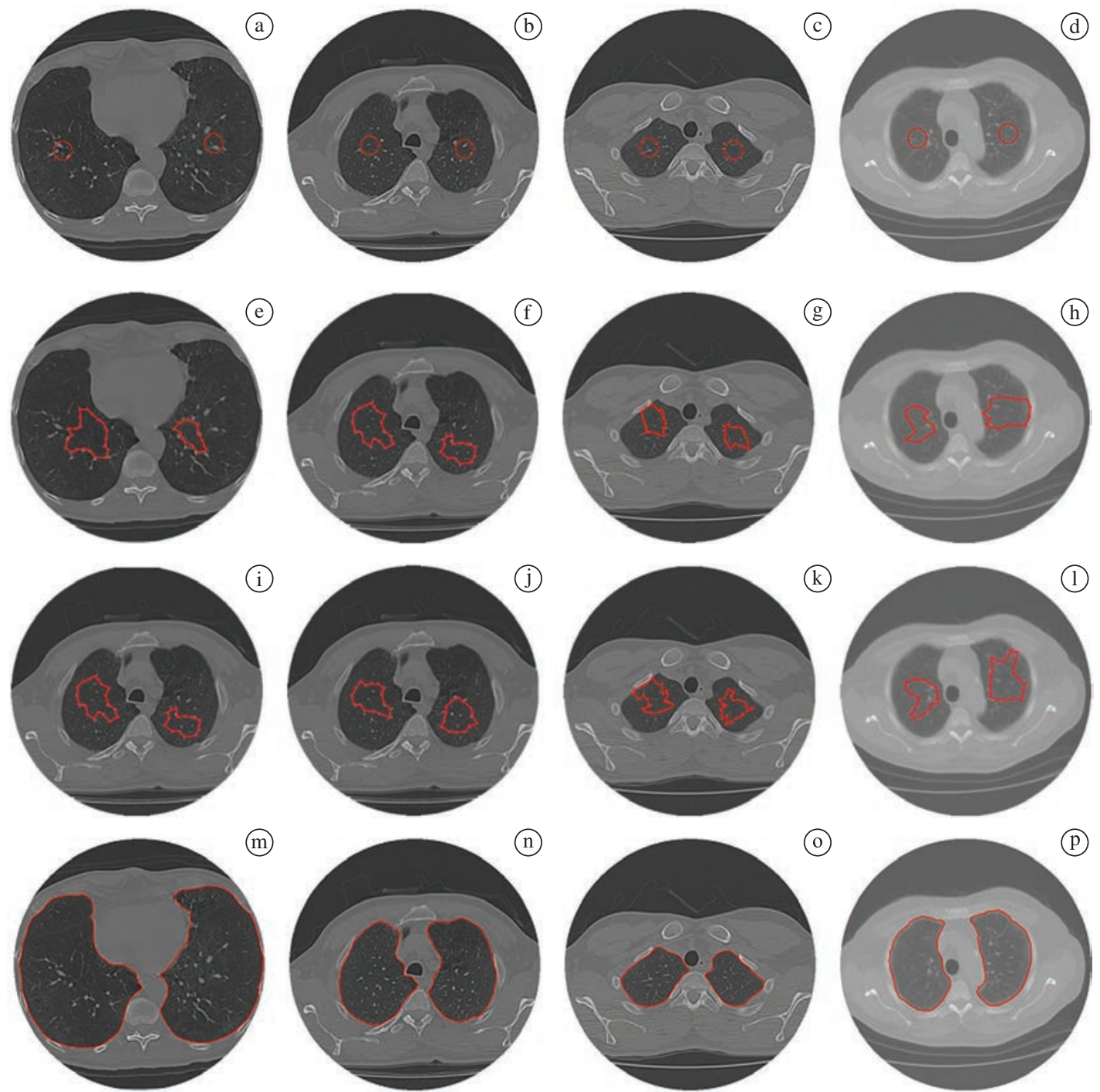

Figura 7. Imagem de TC dos pulmões: a,b,c,d) inicialização da curva, em vermelho, resultados da segmentação aplicando e,f,g,h) MCA Balão, i,j,k,l) MCA GVF, m,n,o,p) MCA Crisp.

Figure 7. CT image of the lungs: $a, b, c, d)$ initiate the curve, red, results of segmentation applying e,f,g,h) ACM balloon, i,j,k,l) ACM GVF, $m, n, o, p)$ ACM Crisp.

Tabela 1. Classificação qualitativa da segmentação dos pulmões. Table 1. Qualitative classification of the segmentation of the lungs.

\begin{tabular}{cl}
\hline Classificação & \multicolumn{1}{c}{ Descrição } \\
\hline Ótimo & $\begin{array}{l}\text { Altíssima qualidade, tão bom quanto } \\
\text { poderia se desejar }\end{array}$ \\
\hline Aceitável & $\begin{array}{l}\text { Qualidade aceitável, com a presença de } \\
\text { pequenos erros }\end{array}$ \\
\hline Razoável & $\begin{array}{l}\text { Média qualidade, com a presença de } \\
\text { erros grosseiros }\end{array}$ \\
\hline Ruim & $\begin{array}{l}\text { Possui apenas uma pequena parcela do } \\
\text { objeto de interesse }\end{array}$ \\
\hline Péssimo & $\begin{array}{l}\text { Não encontra parte nenhuma do objeto } \\
\text { de interesse }\end{array}$ \\
\hline
\end{tabular}

erros de segmentação, sendo considerados como segmentações aceitáveis. As Figuras 8a,b demonstram exemplos de segmentação ótima e aceitáveis em nível de ápice.

Em nível de base encontrados o método proposto obtém $80 \%$ de segmentações ótimas e $20 \%$ com pequenos erros, sendo considerados aceitáveis. Exemplos destes resultados de segmentação são ilustrados nas Figuras 8c,d. Constata-se também que em nível de hilo 33,3\% das segmentações são ótimas, $43 \%$ pequenos erros e $23,7 \%$ erros grosseiros, sendo exemplos destes demonstrados nas Figuras 8e,f. 
Tabela 2. Avaliação qualitativa do método proposto junto ao médico pneumologista.

Table 2. Qualitative assessment of the method proposed by the pulmonologist.

\begin{tabular}{lcccc}
\hline Classificação & Ápice & Base & Hilo & Geral \\
\hline Total & 40 & 30 & 30 & 100 \\
Ótimo & 39 & 24 & 10 & 73 \\
Aceitável & 1 & 6 & 13 & 20 \\
Razoável & 0 & 0 & 7 & 7 \\
Ruim & 0 & 0 & 0 & 0 \\
Péssimo & 0 & 0 & 0 & 0 \\
\hline
\end{tabular}

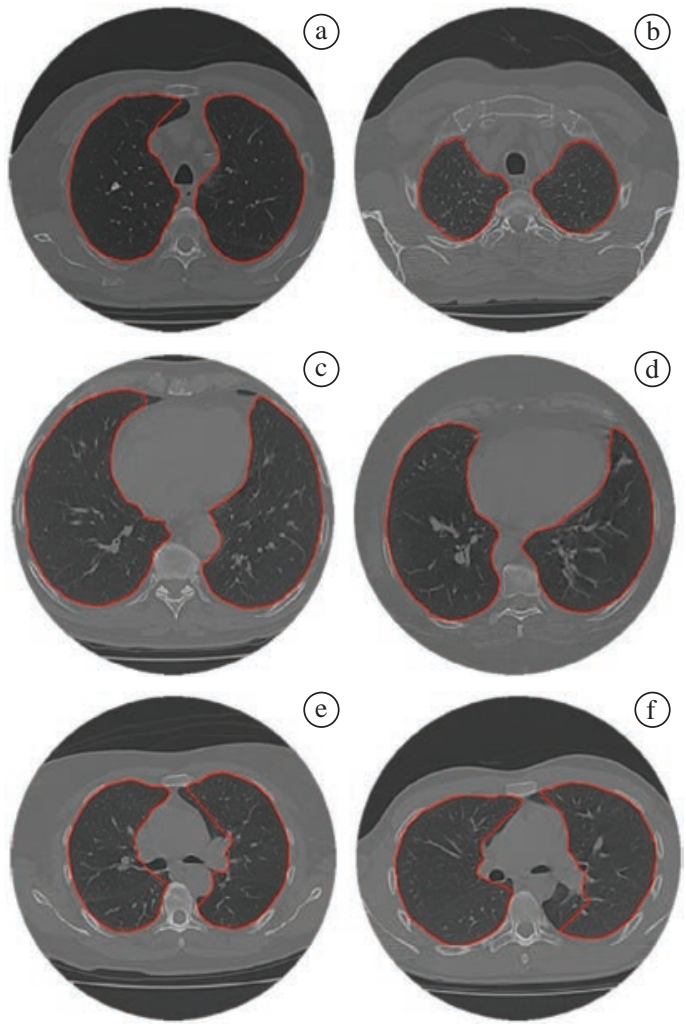

Figura 8. Exemplos de segmentações dos pulmões em imagens de TC pelo método proposto em nível de: a,b) ápice, c,d) base, e,f) hilo. Figure 8. Examples of lungs segmentations in CT images of the method proposed at the level: $a, b)$ apex, c,d) basis, and e,f) hilus.

Considerando todas as imagens analisadas, constatam-se que $73 \%$ das imagens analisadas obtém segmentações ótimas, 20\% segmentações aceitáveis e 7\% de segmentações razoáveis.

\section{Conclusões}

Este trabalho propõe um Método de Contorno Ativo, denominado de MCA Crisp, para a segmentação automática dos pulmões em imagens de TC. Realiza também uma comparação com os MCAs Tradicional,
GVF e Balão, utilizando, para isto, um conjunto de imagens de Tomografia Computadorizada. São usadas imagens de TCAR de voluntários saudáveis e pacientes com DPOC

As segmentações obtidas pelo método proposto foram avaliadas por um medico pneumologista do Hospital Universitário Walter Cantídio da UFC, obtendo 93\% das segmentações obtidas com o MCA Crisp satisfatórios, em que não apresentaram erros ou tiveram erros pequenos, que podem ser corrigidos manualmente pelo próprio médico. Entretanto, os MCAs Tradicional, Balão e GVF apresentam resultados insatisfatórios, obtendo segmentações ruins em todos os pulmões.

Estas segmentações são analisadas em três níveis de corte: ápice, hilo e base. O nível de corte que obtém melhores resultados é o nível de ápice. Isto acontece devido ao fato dos pulmões neste nível não possuírem muitas concavidades e sem muitos vasos sanguineos. Já o nível de corte que obteve os piores resultados é o hilo. Isto acontece devido a irregularidade no formato dos pulmões, com diversos tipos de concavidades neste nível e pela grande quantidade de vasos sanguineos presentes.

Observa-se também que os resultados obtidos pelo método proposto permaneceram semelhantes nas imagens obtidas nos dois tomógrafos utilizados, TCAR e TC com multidetectores.

Desta forma, pode-se afirmar que o Método de Contorno Ativo Crisp é um algoritmo útil para a segmentação dos pulmões, podendo ser integrado a sistemas que auxiliem o diagnóstico médico, visto que segmenta de forma automática e satisfatória os pulmões em imagens de TC, o que diminui o tempo de análise do médico, pois até nas imagens que obtém erros de segmentação é possível corrigir alguns pontos manualmente, sem a necessidade de fazer a segmentação completa, que é uma operação enfadonha e cansativa.

Diversas contribuições são apresentadas neste método, cuja principal é a nova energia externa do método que distingue as bordas das estruturas internas do pulmão das bordas pulmonares. Isto é possível pela análise da vizinhança de cada borda, em que este método determina uma vizinhança ótima de $9 \times 9$ para esta análise.

Outra contribuição é o método de inicialização, obtendo resultados satisfatórios. Além disto, este método descreve métodos de adição e remoção de pontos do contorno, o que não é descrito na literatura nos outros métodos.

A perspectiva nos trabalhos futuros está voltada para dois pontos principais: melhoria da interpretação das falsas bordas pulmonares e estudo de técnicas que auxiliem o contorno a segmentar as regiões côncavas quando o contorno é inicializado internamente. 


\section{Agradecimentos}

Ao CNPq - Conselho Nacional de Pesquisa. Aos Serviços de Pneumologia e de Imagem do Hospital Universitário Walter Cantídio da Universidade Federal do Ceará.

\section{Referências}

Amini A, Weymouth T, Jain RC. Using dynamic programming for solving variational problems in vision. IEEE Transactions on Pattern Analysis and Machine Intelligence. 1990; 12(9):855-67. http://dx.doi. org/10.1109/34.57681

Bouhours A. Segmentação do ventrículo em ecocardiogramas usando contornos ativos [dissertação]. Fortaleza: Universidade Federal do Ceará, 2006.

Cohen LD. On active contour models and balloons. CVGIV: Image understanding. 1991; 53(2):211-8. http://dx.doi. org/10.1016/1049-9660(91)90028-N

Félix JHS. Sistema de visão computacional para detecção e quantificação de enfisema pulmonar [dissertação]. Fortaleza: Universidade Federal do Ceará, 2007.

Fortaleza SCB. Efeitos da administração de pressão positiva contínua em vias aéreas de modo não invasivo sobre a aeração do parênquima pulmonar em pacientes com doença pulmonar obstrutiva crônica [dissertação]. Fortaleza: Universidade Federal do Ceará, 2006.

Gevenois PA, Yernault JC. Can computed tomography quantify pulmonary emphysema? European Respiratory Journal. 1995; 8(5):843-8. PubMed: PMID 7656960.

Gonzales RC, Woods R. Digital image processing. 3th ed. New Jersey: Pearson Prentice Hall, 2008.

Itai Y, Hyoungseop K, Ishikawa S, Katsuragawa S, Ishida T, Nakamura K, Yamamoto A. Automatic segmentation of lung areas based on snakes and extraction of abnormal areas. In: ICTAI05: Proceedings of the 17th IEEE International Conference on Tools with Artificial Intelligence, 2005, Hong
Kong. IEEE, 2005. p. 381-6. http://dx.doi.org/10.1109/ ICTAI.2005.44

Kass M, Witkin A, Terzopoulos D. Snakes: active contours models. International Journal of Computer Vision. 1987. 1(4):321-31. http://dx.doi.org/10.1007/ BF00133570

Mackiewich B. Intracranial boundary detection and radio frequency correction in magnetic resonance images [dissertation]. Burnaby: Simon Fraser University, 1995.

Nixon MS, Aguado AS. Feature extraction and image processing. Woburn: Newnes, 2002.

Sardanelli F, Giuseppetti GM., Panizza P, Bazzocchi M, Fausto A, Simonetti G, Lattanzio V, Maschio AD. Sensitivity of MRI versus mammography for detecting foci of multifocal, multicentric breast cancer in fatty and dense breasts using the whole-breast pathologic examination as a gold standard. American Journal of Roentgenology. 2004; 183(4):1149-57.

Souza Júnior OS. Método de reconhecimento pessoal através da íris usando funções geoestatísticas [dissertação]. São Luiz: Universidade Federal do Maranhão; 2006.

Winkeler GFP. Efeitos agudos da administração de pressão positiva contínua em vias aéreas de modo não invasivo sobre o parênquima pulmonar de voluntários sadios nas posições supina e prona: alterações na tomografia computadorizada de alta resolução [dissertação]. Fortaleza: Universidade Federal do Ceará; 2006. 99p.

$\mathrm{Xu}$ C, Prince J. Snakes, Shapes and gradient vector flow. IEEE Transactions on Image Processing. 1998; 7(3):359-69. http://dx.doi.org/10.1109/83.661186

Zijdenbos AP, Dawant BM, Margolin RA, Palmer AC. Morphometric analysis of white matter lesions in MR images: method and validation. IEEE Transactions on Medical Imaging. 1994; 13(4):716-24. http://dx.doi. org/10.1109/42.363096

Zaidi H, Naqa IE. Pet-guided delineation of radiation therapy treatment volumes: a survey of image segmentation techniques. European Journal of Nuclear Medicine and Molecular Imaging. 2010; 37(11):2165-87. PubMed: PMID 20336455. http://dx.doi.org/10.1007/BF00133570

\footnotetext{
Autores

Pedro Pedrosa Rebouças Filho, Paulo César Cortez

Departamento de Engenharia de Teleinformática, Universidade Federal do Ceará - UFC,

Rua Ana Batista, 510, Jardim Iracema, CEP 60341-360, Fortaleza, CE, Brasil

\section{Marcelo Alcântara Holanda}

Departamento de Pneumologia, Hospital Universitário Walter Cantídio, Universidade Federal do Ceará - UFC, Fortaleza, CE, Brasil
} 